both populations need to be targeted to relieve neuropathic pain.

In irradiated mice transplanted with BM stem cells expressing green fluorescent protein (GFP), it was shown that BM-derived macrophages infiltrate the spinal cord parenchyma and differentiate into highly ramified microglia following partial ligation of the sciatic nerve. Monocyte chemoattractive protein-1 (CCL2, or MCP-1), produced endogenously by injured neurons, induced microglial activation, whereas treatment with a CCL2 neutralizing antibody markedly reduced infiltration of GFP-positive cells to the parenchyma. In mice deficient for the CCL2 receptor, CCR2, microglial activation did not occur following injury, and hypersensitivity to mechanical stimuli was attenuated in these mice compared with controls $(P<0.05)$. However, hypersensitivity was not decreased by selective knockout of CCR2 on either CNS-resident or BM-derived microglia alone.

The authors conclude that both CNS-resident and BM-derived microglia need to be targeted to relieve neuropathic pain. They also suggest that the selective migration of BM-derived macrophages to affected CNS regions presents the possibility that these cells could be used as drug-delivery vehicles for pain relief.

Original article Zhang J et al. (2007) Expression of CCR2 in both resident and bone marrow-derived microglia plays a critical role in neuropathic pain. J Neurosci 27: 12396-12406

\section{Heterogeneity in cortical surface shape of patients with autism spectrum disorders}

Evidence from neuroimaging is shedding light on the neuropathology of autism. A recent study by Nordahl et al. has characterized cortical folding patterns in subgroups of autism spectrum disorders by use of high-resolution threedimensional T1-weighted MRI to generate maps of cortical surfaces and sulcal depth.

The researchers compared cortical shape in males aged 7.5-18.5 years with low-functioning autism (LFA; $n=17$ ), high-functioning autism (HFA; $n=14$ ), or Asperger's syndrome (ASP; $n=15$ ) with that in typically developing controls (TD; $n=29$ ), and revealed folding abnormalities in each autism spectrum subgroup. Sulcal depth in the left frontal operculum and anterior insula was significantly greater in the LFA group than in the TD group $(P=0.01)$. This finding was accounted for by a shape abnormality localized to the pars opercularis in the LFA group. Sulcal depth in the bilateral parietal operculum was greater in the HFA group than in the TD group $(P=0.01)$. A bilateral shape irregularity was apparent in the intraparietal sulcus in the ASP group, an abnormality that correlated with the participants' age, IQ, and performance on a measure of autism behavior symptoms. Separate analysis by age-group revealed that the differences in the LFA and ASP groups were more pronounced in children (aged 7.512.5 years) than in adolescents (aged 12.7518.5 years). The authors suggest that the results of the study support the idea that the course of early brain development is altered in autism.

Further studies are needed to elucidate the functional significance of the anatomical differences observed among the subgroups of autism spectrum disorders.

Original article Nordahl CW et al. (2007) Cortical folding abnormalities in autism revealed by surface-based morphometry. J Neurosci 27: 11725-11735

\section{Can NSAIDs protect against Parkinson's disease in humans?}

Growing evidence indicates that neuroinflammatory processes contribute to cell death in Parkinson's disease (PD). Numerous animal studies have suggested that NSAIDs can have a neuroprotective effect in PD, but to date few human studies have investigated this possibility. To address this issue, Wahner et al. performed a population-based study to assess the use of NSAIDs in 293 patients with idiopathic PD and 286 controls matched for age, race and sex.

The results suggested that regular NSAID users who took more than two pills per week for at least 1 month had a decreased risk of developing PD compared with controls. Regular users of nonaspirin NSAIDs, particularly those who took pills regularly over at least 2 years, showed the greatest decrease in risk. Aspirin use had a significant neuroprotective effect in women only, but the authors note that this finding could represent a dose-related effect; men are more likely to take aspirin at children's doses for prevention of cardiovascular events, whereas women probably take aspirin in larger doses for pain relief. 